\title{
Aleación superficial de superaleaciones base níquel mediante láser
}

\author{
G. P. Rodríguez ${ }^{(*)}$, I. García ${ }^{(* *)}$ y J.J. de Damborenea ${ }^{(* *)}$
}

\begin{abstract}
Resumen: Las superaleaciones base níquel presentan una elevada resistencia a la oxidación a alta temperatura. así como excelentes propiedades mecánicas. Los nuevos desarrollos tecnológicos (condiciones cada vez más agresivas) obligan a una casi continua modificación de dichas aleaciones. En el presente trabajo, dos superaleaciones base níquel (Nimonic 80A e Inconel 600) se alean superficialmente con aluminio utilizando un láser de alta potencia. Se estudia la microestructura de la capas obtenidas mediante SEM y EDX. Se realizan ensayos de oxidación a $1.273 \mathrm{~K}$ sobre las probetas aleadas y sobre el material base durante tiempos variables entre 24 y $250 \mathrm{~h}$. Los resultados indican la formación de una capa protectora de alúmina sobre las probetas aleadas que puede incrementar la resistencia a la oxidación.
\end{abstract}

Palabras clave: Aleación láser. Oxidación. Superaleaciones.

\section{Surface alloying of nickel based superalloys by laser}

\begin{abstract}
Ni based superalloys present a high oxidation resistance at high temperature as well as good mechanical properties. But new tecnhology developments force to research in this materials to improve their properties at high temperature. In this work, two Ni based superalloys (Nimonic 80A and Inconel 600) were surface alloyed with aluminium using a high power laser. SEM and EDX were used to study the microstructure of the obtained coatings. Alloyed specimens were tested at $1.273 \mathrm{~K}$ between 24 and $250 \mathrm{~h}$. Results showed the generation of a protective and continuous coating of alumina on the laser treated specimens surface that can improve oxidation resistance.
\end{abstract}

Keywords: Laser treatments. Oxidation. Superalloys.

\section{INTRODUCCIÓN}

Las superaleaciones base níquel presentan alta resistencia mecánica a elevada temperatura, alta resistencia a la fluencia, a la fatiga térmica y a la erosión, y sus altos puntos de fusión previenen, en el caso de sobrecalentamientos accidentales, de fusiones incipientes. Además, poseen una excelente resistencia a la oxidación, lo que hace que sean excelentes candidatas en aplicaciones en las que se requiera trabajar a temperaturas elevadas $(>1.000 \mathrm{~K})$, como son las turbinas o los sistemas de conversión de energía ( 1 y 2). Debido a que, en la mayoría de los medios, está presente el oxígeno, la oxidación es el mecanismo de degradación más importante a altas temperaturas. La resistencia a la oxidación dependerá fundamentalmente de la capacidad de las aleacio-

(*) ETSII, Univ. de Castilla-La Mancha. Ciudad Real (España).

(**) Centro Nacional de Investigaciones Metalúrgicas, CENIM (CSIC). Madrid (España). nes para formar y mantener una capa protectora de óxidos con bajas velocidades de crecimiento. El papel de esta capa es aislar la aleación base del medio agresivo y así limitar el ataque por corrosión.

Uno de los métodos más utilizados para mejorar la resistencia a la oxidación de las superaleaciones base níquel consiste en aumentar el contenido superficial de aluminio con el fin de obtener una capa externa formada por compuestos de níquel y aluminio (3 y 4). Las fases $\mathrm{NiAl}, \mathrm{Ni}_{3} \mathrm{Al}$ e, incluso $\mathrm{NiAl}_{3}$, además de tener elevados puntos de fusión, se oxidan lentamente dando lugar a capas protectoras de $\mathrm{Al}_{2} \mathrm{O}_{3}$.

En este trabajo se estudia la producción mediante láser de una aleación superficial de aluminio sobre las superaleaciones Inconel 600 y Nimonic 80A. Se realizó un estudio de la incorporación de aluminio a la superficie a través de la morfología y análisis de composición mediante SEM y EDX para distintos parámetros del procesado láser. Las probetas así obtenidas se expusieron en atmósferas a 
1.273 K durante períodos de hasta 250 h, estudiándose los cambios morfológicos aparecidos como consecuencia del proceso.

\section{PROCEDIMIENTO EXPERIMENTAL}

La composición inicial de las aleaciones objeto de estudio es la que se presenta a continuación:

Inconel 600: Ni 72,89, Cr 16,64, Fe 8,89, Co 0,49, Si $0,47, \%$ en masa.

Nimonic 80A: Ni 74,6, Cr 19,58, Ti 2,52, Al 1,46, $\mathrm{Fe} 0,77$, Co 0,26, \% en masa.

Las probetas utilizadas procedían de un redondo de $30 \mathrm{~mm}$ (Nimonic) y $60 \mathrm{~mm}$ (Inconel) de diámetro. Previamente al tratamiento con láser, se granalló la superficie para reducir las perdidas por reflexión del haz láser, obteniéndose un parámetro $R a$ de rugosidad de $5 \mu \mathrm{m}$ en ambos casos. Se empleo un láser continuo de $\mathrm{CO}_{2}$ (Spectra Physics) que trabajaba en haz multimodo, con una potencia de salida de $5 \mathrm{~kW}$ y un área sobre la superficie del material de $64 \mathrm{~mm}^{2}$. El haz láser barría la superficie de las probetas para formar los cordones mediante el movimiento a velocidad constante de estas. El proceso se realizó con una velocidad de barrido de $500 \mathrm{~mm} / \mathrm{min}$ con protección gaseosa de $45 \mathrm{~L} / \mathrm{min}$ de argón.

La aleación se realizó utilizando la técnica de inyección de partículas. Para ello, se dirige un caudal determinado $(3 \mathrm{~g} / \mathrm{min})$ de polvo de aluminio de alta pureza $(99,9 \%)$ contra la superficie de la probeta. Cuando el haz láser se focaliza sobre la misma, intercepta de manera casi simultánea al aluminio y a la aleación de níquel, produciéndose la interdifusión del aleante con el material base (5).

Se realizaron ensayos de oxidación a altas temperaturas introduciendo las probetas en un horno previamente calentado a $1.273 \mathrm{~K}$ en atmósfera de aire y manteniendo el material a esta temperatura durante tiempos variables entre 24 y $250 \mathrm{~h}$. Una vez tratadas en horno, las probetas se cortaron en sección transversal y se preparan metalográficamente. Las microestructuras obtenidas tras la aleación con láser, así como las resultantes tras el tratamiento térmico se estudiaron mediante microscopía electrónica de barrido y se realizaron análisis EDX para identificar las distintas fases presentes.

\section{RESULTADOS Y DISCUSIÓN}

La estructura metalográfica de ambos materiales está formada por una solución sólida de níquel $(\gamma)$ con precipitados de distintos compuestos intermetá- licos. La formación de precipitados coherentes en la matriz ( proporciona el endurecimiento de estas aleaciones. La microestructura del material base es equiaxial con un tamaño de grano, similar en ambas aleaciones, de 5.5 ASTM.

Sobre cada probeta se realizaron tres cordones en las mismas condiciones. Todos los cordones presentan buen aspecto, sin falta de adherencia o defectos superficiales (falta de fusión), tal y como se aprecia en la figura 1 para el caso del Nimonic. La rugosidad media de los cordones, $R a$, es de 4,9 $\mu \mathrm{m}$ para el caso del Nimonic y de $6 \mu \mathrm{m}$ para el Inconel. Ambos valores pueden considerarse del mismo orden que la del material de partida, $5 \mu \mathrm{m}$.

En algunos casos, se aprecia la formación de grietas originadas fundamentalmente en el centro
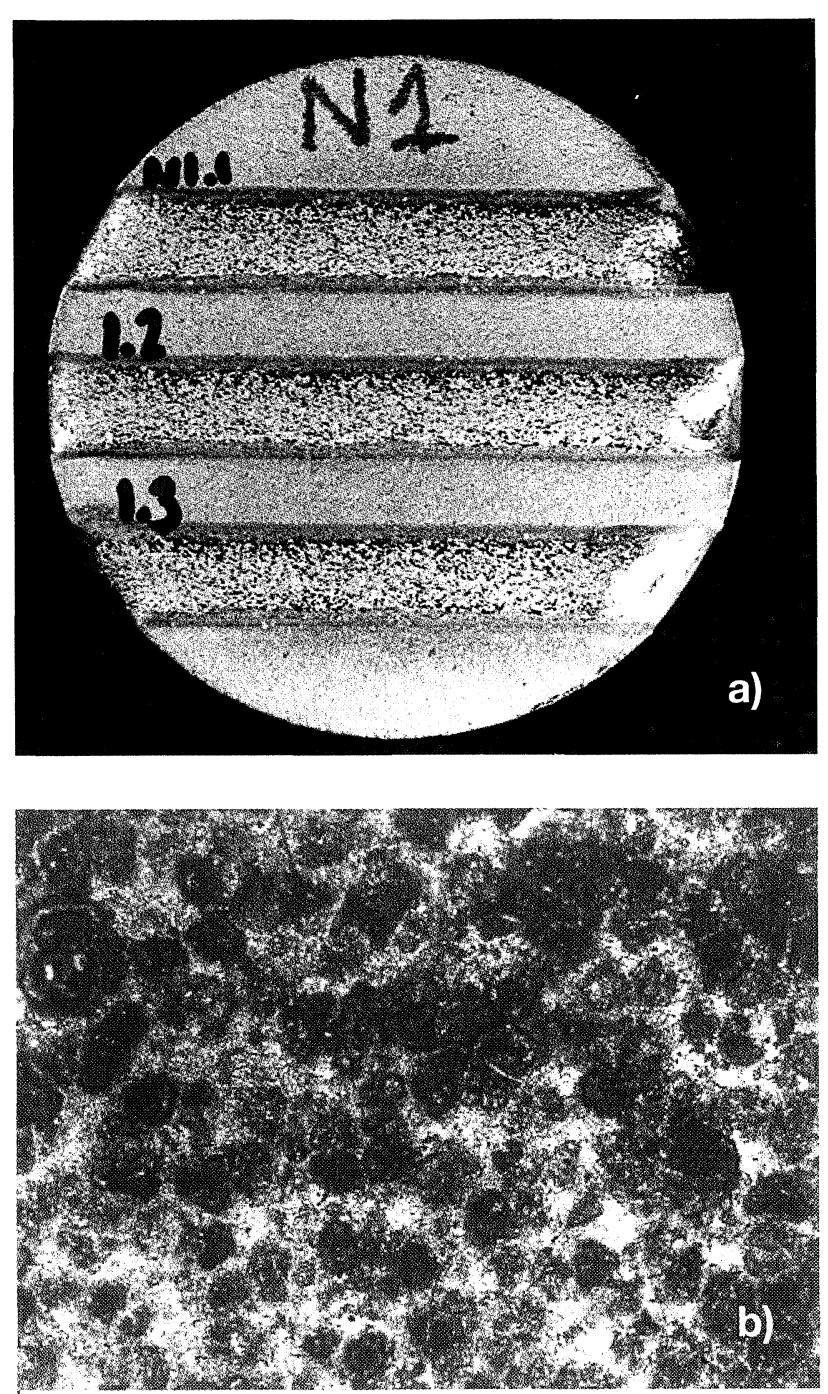

FIG. 1 a).- Macrografía de los cordones realizados sobre el Nimonic. $b$ ) Micrografía de la superficie del cordón realizado sobre Inconel. $\times 50$.

FIG. 1 a).-Macrography of laser tracks on Nimonic. b) Micrography of the surface of a laser track on Inconel $\times 50$. 
de los cordones, si bien no aparecen en el resto del cordón, ni en la zona afectada térmicamente, ni en la interfase cordón-material base. La formación de grietas en el centro de los cordones es consecuencia de los fenómenos de contracción asociados con la solidificación.

En la figura 2 se muestran cortes transversales de los cordones producidos a la velocidad elegida. En ellos se aprecian tres zonas diferenciadas entre la superficie del cordón y la intercara metal/recubrimiento.

En la observación microscópica se distinguen tres zonas en la estructura del cordón (Fig. $3 a$ y $b$ ). Una zona interna, cercana al material base, formada fundamentalmente por una solución sólida de níquel $\gamma$. La zona central presenta una estructura bifásica formada por precipitados del compuesto intermetálico $\mathrm{Ni}_{3} \mathrm{Al}\left(\gamma^{\prime}\right)$ en una matriz $\gamma$. Finalmente, una zona superficial formada mayoritariamente por compuestos intermetálicos del tipo $\mathrm{Ni}_{3} \mathrm{Al}$. $\mathrm{La}$
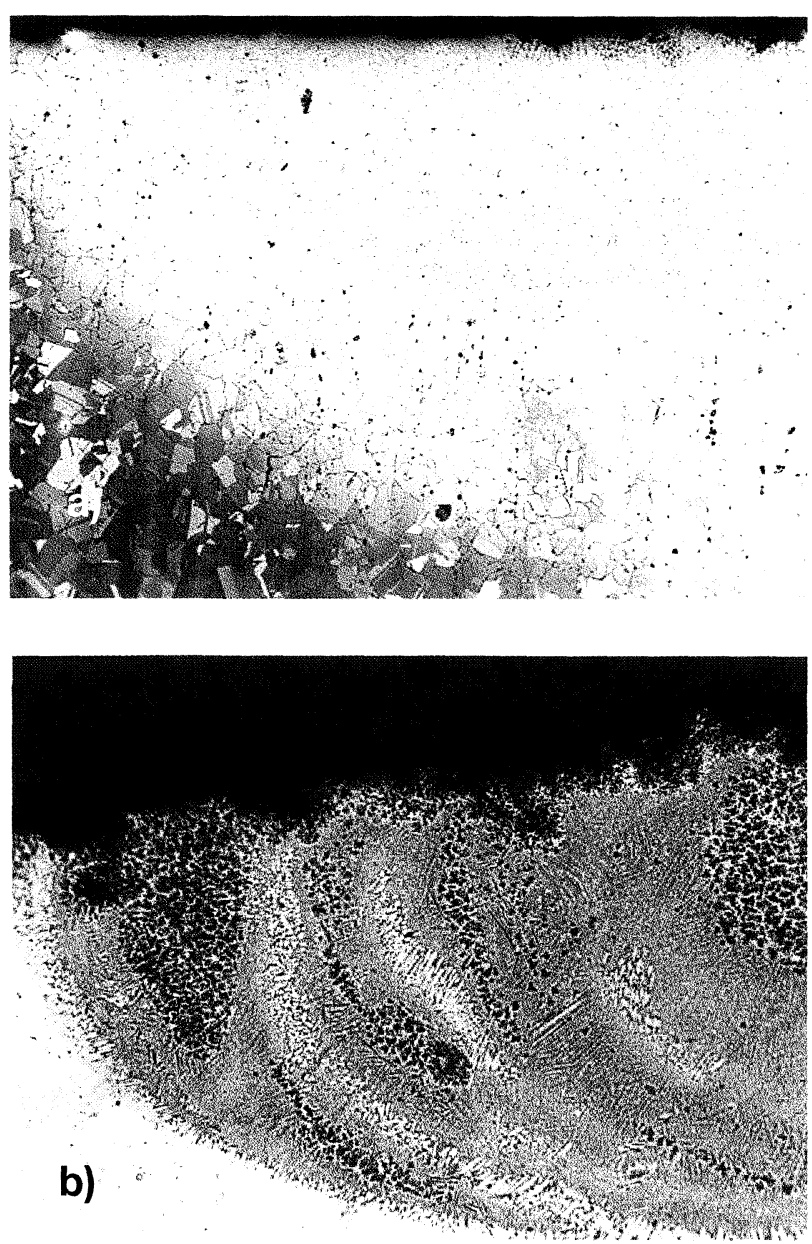

FIG. 2.- Micrografías de cortes transversales de cordones procesados a la velocidad de $500 \mathrm{~mm} / \mathrm{min}$.

a) Nimonic, $b$ ) Inconel. $\times 100$.

FIG. 2.- Micrographies of tranversal view of the laser tracks obtained at $500 \mathrm{~mm} / \mathrm{min}$. a) Nimonic b) Inconel. $\times 100$.
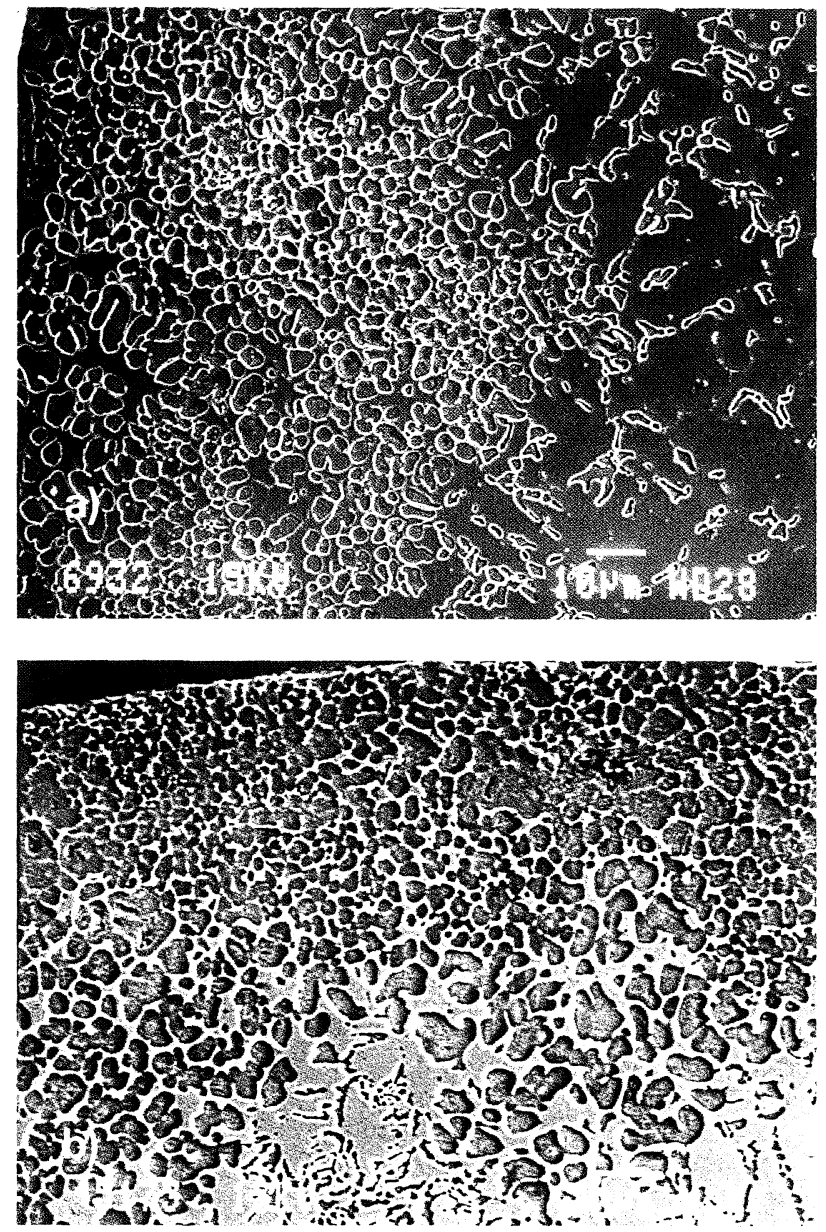

FIG. 3.- Imágenes SEM de las zonas superficiales de los cordones. $a$ ) Nimonic, $b$ ) Inconel.

FIG. 3.- SEM images of the surface of the laser tracks. a) Nimonic. b) Inconel.

baja densidad del aluminio hace que su concentración sea mayor en la superficie, lo que justifica que la presencia de $\gamma^{\prime}$ vaya disminuyendo con la profundidad en el cordón. La composición del compuesto intermetálico $\gamma^{\prime} \mathrm{Ni}_{3} \mathrm{Al}$ no corresponde exactamente con esta estequiometría, puesto que parte del níquel es desplazado por cromo. Damborenea et al. (6) justifican este hecho debido a que la rápida solidificación obtenida con el tratamiento con láser conduce a la formación de fases metaestables con concentraciones distintas a las indicadas en el diagrama Ni-Cr-Al.

Los ensayos de oxidación a $1.273 \mathrm{~K}$ revelan un mejor comportamiento del material aleado que del material sin alear. En la figura 4 se muestra el aspecto de la capa de óxido formada sobre el Inconel $(a)$ y sobre el Nimonic $(b)$ sin alear después de permanecer $100 \mathrm{~h}$ a $1.000{ }^{\circ} \mathrm{C}$ en atmósfera de aire. Puede apreciarse el crecimiento de una capa de óxido con presencia de poros y aspecto no adherente. Su composición, en el caso del Inconel, es de $\mathrm{Cr}$ $65 \%$, Ni $22 \%$, Si $6 \%$, Fe $4 \%$ y Ti $1 \%$. Es decir, 

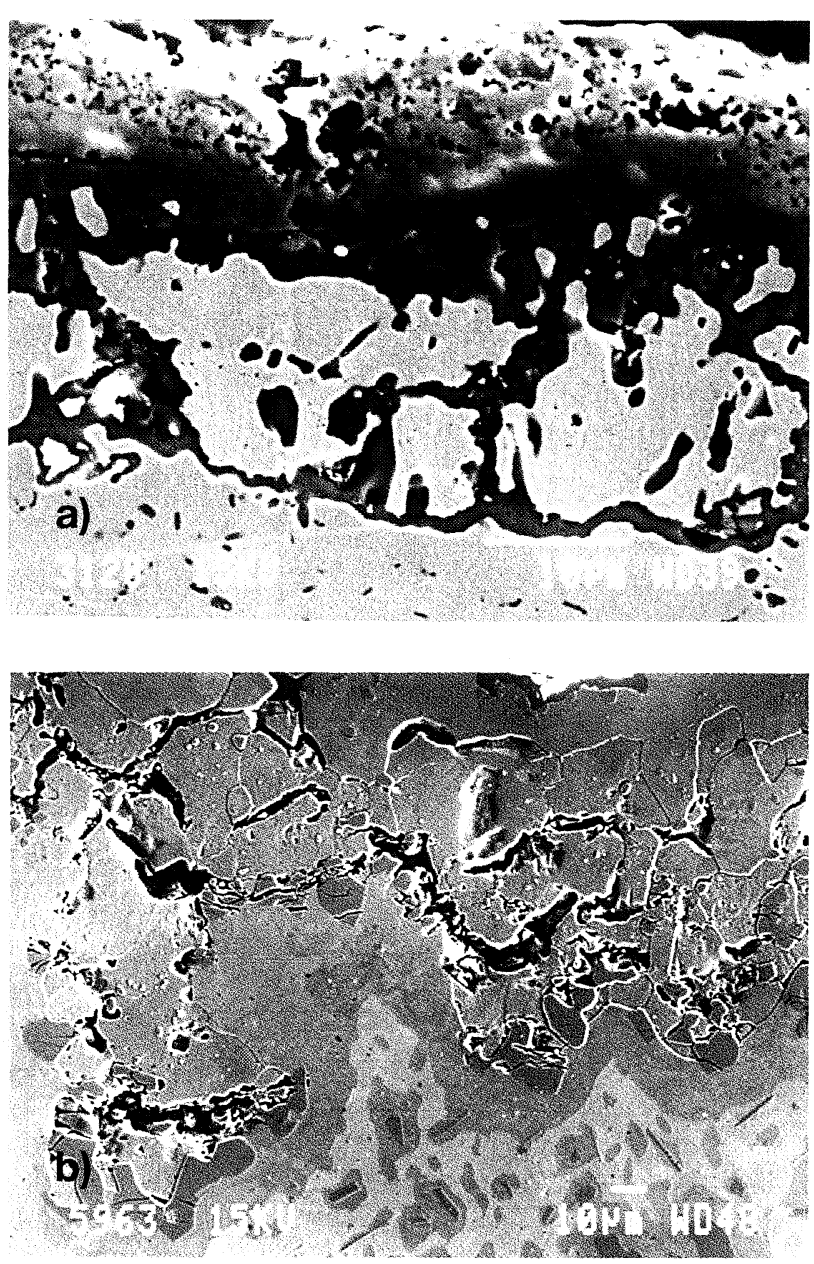

FIG. 4.- Imágenes SEM de la superficie de los materiales sin alear sometidas durante $100 \mathrm{~h}$ a 1.273

$\mathrm{K}$ en atmósfera de aire. a) Nimonic, b) Inconel.

FIG. 4.- SEM images of the base materials after $100 \mathrm{~h}$ at $1.273 \mathrm{~K}$ in air. a) Nimonic. b) Inconel.

está formada mayoritariamente por óxidos de cromo más $\mathrm{NiO}$ y $\mathrm{SiO}_{2}$. Sobre las probetas de Nimonic también se observa la formación de una capa de óxido no protectora, así como un ataque intergranular desde la superficie al interior del material. El análisis de la capa de óxido muestra una primera capa más externa de $\mathrm{NiO}$, a continuación una capa de óxidos de níquel y cromo, y una capa más interna en contacto con el material base de óxidos de cromo y titanio. El espesor de la capa de óxidos aumenta considerablemente con el tiempo de tratamiento (espesores superiores a $25 \mathrm{~mm}$ cuando la oxidación se produce durante $250 \mathrm{~h}$ frente a espesores de $8 \mathrm{~mm}$ en tratamientos de $24 \mathrm{~h}$ ). Asimismo, el ataque en el interior del material es mucho más pronunciado cuando los tiempos de calentamiento son mayores.

La oxidación de las probetas previamente aleadas con láser conduce, para ambas superaleaciones, a la formación de una capa continua y protectora de
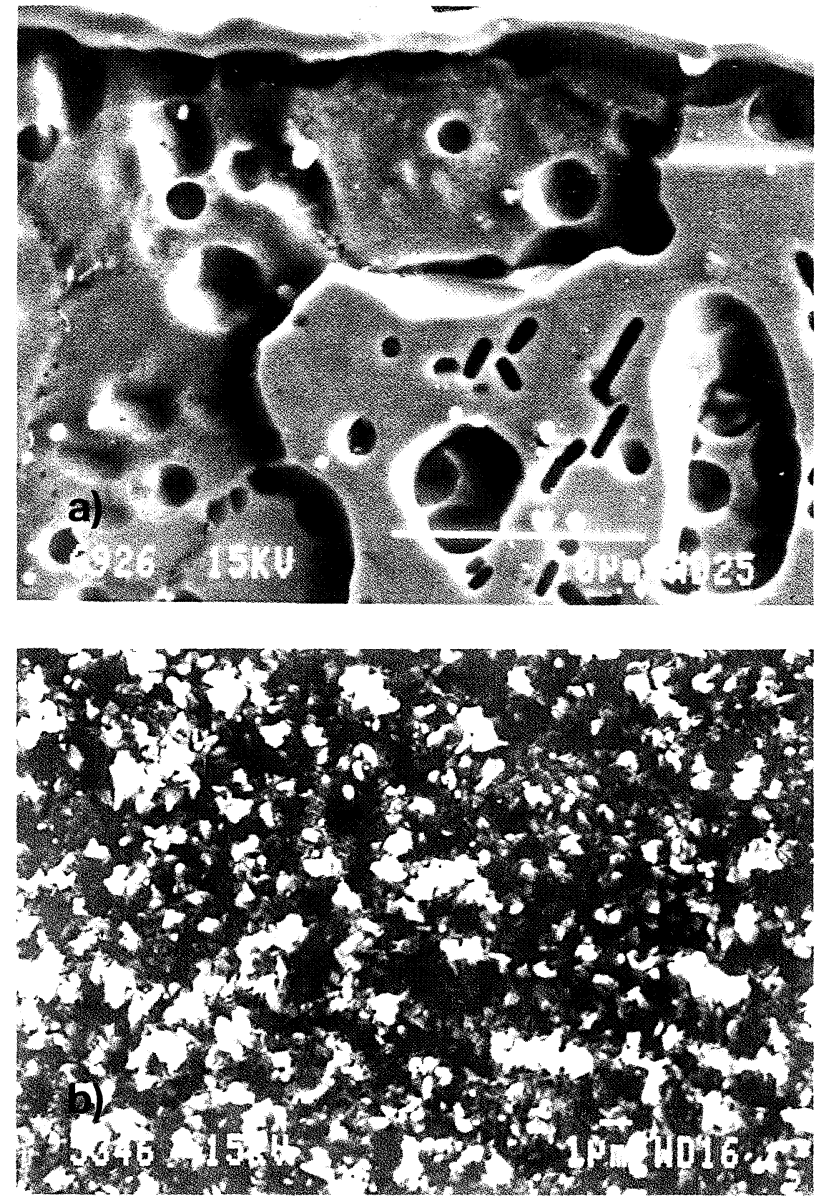

FIG. 5.- Imagen SEM del corte transversal de la superficie de las probetas de Nimonic tratadas con láser después de oxidarse a $1.273 \mathrm{~K}$ a) Capa de alúmina generada después de 50 h. $b$ )Tras 100 h de exposición.

FIG. 5.- SEM image of Nimonic laser treated surface at $1.273 \mathrm{~K}$ a) Alumina coating generated after $50 \mathrm{~h}$. b) Nimonic after $100 \mathrm{~h}$.

alúmina en superficie que actúa como barrera al paso del oxígeno, de manera que no se observa ningún ataque en el interior del material. En la figura 5 $a$ y $b$ se observa esta situación junto con la de la formación de la capa de alúmina. Su composición, que no varía apreciablemente con el tiempo de oxidación a alta temperatura, es de $\mathrm{Al}$ 83-88 \%, Cr 8$11 \%$, Ni $3-4 \%$ y $\mathrm{Fe} 1 \%$. Por tanto, la capa está formada por $\mathrm{Al}_{2} \mathrm{O}_{3}$ más una pequeña presencia de óxidos de cromo y níquel provenientes, posiblemente, de la capa de óxido de transición formada en los primeros instantes de oxidación.

Como consecuencia del tratamiento, se pone de manifiesto un segundo efecto: en la zona superficial del cordón, justo por debajo de la capa de óxido, en lugar de aparecer la microestructura bifásica $(\gamma+$ $\left.\gamma^{\prime}\right)$, presente en el cordón antes del tratamiento, se observa una tercera fase enriquecida en cromo (fase ( que consiste en una solución sólida de cromo) que presenta un aspecto globular. La aparición de esta 
fase es consecuencia de los procesos de difusión, activados al aumentar la temperatura según $\gamma+\gamma^{\prime}=$ $\gamma+\gamma+\gamma^{\prime}(7)$

La superficie de los cordones muestra zonas donde se ha desprendido la capa de alúmina. Esta pérdida de la capa se ha producido durante el enfriamiento debido a las tensiones térmicas, como se demuestra por el hecho de que la composición del material expuesto en estas zonas no presenta óxidos de cromo (Ni $64 \%, \mathrm{Cr} 13 \%, \mathrm{Fe} 10 \%$ y Al $5 \%$ ). Este efecto, que puede ser perjudicial para el empleo de las superaleaciones aleadas superficialmente con aluminio en aplicaciones con ciclos térmicos, aunque puede corregirse con la incorporación de elementos de transición, como el hafnio o el ytrio, cuya incorporación a la superficie mediante láser ya ha sido objeto de estudio con buenos resultados (8).

\section{CONCLUSIONES}

La aleación superficial con láser de aluminio sobre la superaleación Inconel 600 y Nimonic 80A forma una capa homogénea de compuestos intermetálicos $\gamma^{\prime}\left(\mathrm{Ni}_{3} \mathrm{Al}\right)$ en una matriz de solución sólida de níquel $\gamma$. Esta capa aleada es continua, con baja rugosidad y adherente. El comportamiento del material aleado frente a la oxidación a altas temperaturas es notablemente mejor que el de la aleación base. Se forma una capa continua protectora de $\mathrm{Al}_{2} \mathrm{O}_{3}$ que dificulta el acceso de oxígeno al material base y que evita tanto la formación de óxidos no adherentes $\mathrm{NiO}$, como el ataque intergranular en el interior observado en la oxidación del material sin alear.

\section{REFERENCIAS}

(1) Sims, C.T., Stollof, W.S. y Hagel, W.C. (eds.), Superalloys II, John Wiley \& Sons, Nueva York, 1987.

(2) Gonzalez Carrasco, J.L., Adeva, P. y Aballe, M. Oxid. Met. 33 (1990) 1.

(3) Abboud, J.H., West, D.R.F. y Rawlings, R.D. Mater. Sci. Technol. 70 (1994) 848.

(4) Zhu, S., WANG, F., Lou, H. y Wu, W. Surf. Coat. Technol. 71 (1995) 9.

(5) Damborenea, J.J. de, y Vazquez, A.J. J. Mater. Sci. 27 (1992) 1.27.1.

(6) Damborenea, J.J. De, Lopez, V. y Vazqúez, A.J. Surf. Coat. Technol., 70 (1994) 107.

(7) Merchant, S.M. y Notis, M.R. Mater. Sci. Eng. 66 (1984) 47.

(8) Singh, J. y Mazumder, J. Acta. Metall. 35 (1987) 1995. 